\title{
Quantitative assessment of global and regional strain in relation to infarct size in patients with myocardial infarction
}

\author{
Nabil A Shafi ${ }^{*}$, Kathleen Bertman ${ }^{1}$, Andrew Yoon ${ }^{1}$, Rena Toole ${ }^{1}$, Matthew Ronin', Simcha Pollack², \\ Nathaniel Reichek ${ }^{3}$, Madhavi Kadiyala ${ }^{1}$
}

From 2011 SCMR/Euro CMR Joint Scientific Sessions

Nice, France. 3-6 February 2011

\section{Introduction}

Assessment of regional and global left ventricular (LV) function and strain are important in the setting of prior myocardial infarction (MI). Feature tracking (FT-MRI) is a novel cardiac MRI method for assessment of myocardial strain, similar to speckle tracking in 2-D echocardiography, which provides multi-planar strain data without the need for tagged images.

\section{Purpose}

The goal of our study was to characterize LV global and regional strains in patients with prior MI using FT-MRI and determine the relationship of strains to infarct size, location and LV ejection fraction (EF).

\section{Methods}

Eighty patients with history of past MI were grouped by MI coronary territory \{left anterior descending (LAD), left circumflex (LCX), right coronary (RCA)\}. Scar quantification was done by computer-assisted planimetry (Medis Qmass v7.2) of gadolinium enhanced delayed images and MI percentage was determined. Global and regional circumferential subendocardial (Ecc Endo) and subepicardial (Ecc Epi) and longitudinal (Ell) strains were derived from three long axis (4,3 and 2 chamber) and three short axis (basal, mid, apical) steady state free procession cine planes. Semi-automated tracing of endocardial and epicardial borders using FT-MRI (Diogenes MRI, Tomtec Systems) was performed and strains were mapped to a 17 segment AHA model. Repeated measures ANOVA was used to compare normal strain values from 60 healthy subjects to MI patients controlling for scar percentage.

\section{Results}

The mean age was 64.4(12) years and mean EF was 42.1\% (11). Peak global Ecc Epi and Ecc Endo were decreased in all MI patients, except in Ecc endo LCx infarcts, compared to normals (all with $\mathrm{p} \leq 0.01$ ) (Table 1). Peak Ell strain was relatively preserved in the majority of infarcts except in LAD infarcts, where it was significantly decreased in the LAD territory ( $\mathrm{p}=0.01$ ). The best predictor of reduced LVEF in MI patients was reduced global Ecc Endo $(r=0.82, p<0.0001)$, followed by global Ecc Epi $(r=0.64, p<0.0001)$, scar territory percent $(r=0.53$, $\mathrm{p}<0.0001)$ and global Ell $(\mathrm{r}=0.43, \mathrm{p}<0.0001)$.

\section{Conclusion}

FT-MRI permits detailed assessment of global and regional strains in patients with MI. Global Ecc was decreased in all MI patients, while Ell was preserved in the majority on infarcts except LAD infarcts. The best predictor of reduced LV EF was reduced global Ecc Endo strain, which was superior to infarct size.

\section{Author details \\ ${ }^{1}$ St. Francis Hospital- The Heart Center, Roslyn, NY, USA. ${ }^{2}$ St. Francis Hospital- The Heart Center, Roslyn, NY and, St. John's University, New York City, NY, USA. ${ }^{3}$ St. Francis Hospital- The Heart Center, Roslyn, NY and, Stony Brook University School of Medicine, NY, USA.}

Published: 2 February 2011

${ }^{1}$ St. Francis Hospital- The Heart Center, Roslyn, NY, USA

Full list of author information is available at the end of the article

(c) 2011 Shafi et al; licensee BioMed Central Ltd. This is an open access article distributed under the terms of the Creative Commons 
Table 1

\begin{tabular}{|c|c|c|c|c|c|}
\hline $\begin{array}{l}\text { Patient Demographics and Peak Strain } \\
\text { Values }\end{array}$ & $\begin{array}{l}\text { Normals } \\
(\mathrm{N}=60)\end{array}$ & $\begin{array}{l}\text { LAD Infarct } \\
(\mathrm{N}=22)\end{array}$ & $\begin{array}{l}\text { LCx Infarct } \\
(\mathrm{N}=11)\end{array}$ & $\begin{array}{l}\text { RCA Infarct } \\
(\mathrm{N}=31)\end{array}$ & $\begin{array}{l}\text { Multi infarct } \\
(\mathrm{N}=16)\end{array}$ \\
\hline Age (years) & $54.4(14)$ & $61.9(10)$ & $63.2(9)$ & $62.7(15)$ & $70.0(7)$ \\
\hline LVEF (\%) & $58.2(5)$ & $38(8)$ & $50.1(10)$ & $43.9(12)$ & $36(11)$ \\
\hline Scar (\%) & NA & $23.1(11)$ & $10.2 \pm(6)$ & $14(7)$ & $19 \pm(8)$ \\
\hline \multicolumn{6}{|l|}{ Ell Avg (SD) } \\
\hline Global & $-16.2(5)$ & -9.4 (3) $\mathbf{P}=\mathbf{0 . 0 4}$ & $-10.2(5)$ & $-14.7(5)$ & $-8.6(4)$ \\
\hline Basal & $-14.0(8)$ & $-16.1(5)$ & $-8.1(7)$ & $-12(6)$ & $-8(6)$ \\
\hline Mid & $-14.1(8)$ & $-10.6(3)$ & $-6.7(5)$ & $-11.4(6)$ & $-7.1(5)$ \\
\hline Apical & $-17.1(8)$ & -5.7 (2) $\mathbf{P}=\mathbf{0 . 0 1}$ & $-13.0(4)$ & $-16.5(6)$ & $-9.2(5)$ \\
\hline$\angle A D$ & $-18.8(6)$ & -8.8 (3) $\mathbf{P}=\mathbf{0 . 0 1}$ & $-14.2(5)$ & $-17.6(5)$ & $-9.7(4)$ \\
\hline$L C x$ & $-18.7(8)$ & $-10.0(5)$ & $-12.6(6)$ & $-19(7)$ & $-10(5)$ \\
\hline RCA & $-12.2(10)$ & $-10.8(5)$ & $-5.1(4)$ & $-8.5(7)$ & $-6.6(5)$ \\
\hline \multicolumn{6}{|l|}{ Ecc Epi } \\
\hline Global & $-16.1(3)$ & -8.4 (3) $\mathbf{P}=\mathbf{0 . 0 0 1}$ & -10.6 (3) $\mathbf{P}=\mathbf{0 . 0 1}$ & -9.6 (4) $P=\mathbf{0 . 0 0 1}$ & -7.8 (3) $\mathbf{P}=\mathbf{0 . 0 2 6}$ \\
\hline Basal & $-14.8(3)$ & -10.4 (3) $\mathbf{P}=\mathbf{0 . 0 0 6}$ & $-10.3(3)$ & -8.2 (4) $P=0.006$ & -7.7 (3) $\mathbf{P}=\mathbf{0 . 0 0 4}$ \\
\hline Mid & $-15.6(4)$ & $-8.7(3)$ & -8.1 (3) $\mathbf{P}=\mathbf{0 . 0 0 2}$ & -7.8 (4) $\mathrm{P}<0.001$ & -7.1 (3) $\mathbf{P}<\mathbf{0 . 0 0 1}$ \\
\hline Apical & $-19.3(5)$ & -6.9 (4) $P<\mathbf{0 . 0 0 4}$ & $-14.9(5)$ & -15.8 (8) $\mathbf{P}=\mathbf{0 . 0 0 2}$ & $-9.5(7)$ \\
\hline$\angle A D$ & $-17.1(5)$ & -6.6 (3) $P=\mathbf{0 . 0 0 7}$ & $-13.2(3)$ & -11.9 (6) $\mathbf{P}=\mathbf{0 . 0 0 2}$ & $-8.1(4)$ \\
\hline$L C x$ & $-22.5(7)$ & -13.3 (6) $\mathbf{P}=\mathbf{0 . 0 3}$ & $-10.9(£) \mathbf{P}=\mathbf{0 . 0 2}$ & -15.5 (7) $\mathbf{P}=\mathbf{0 . 0 1}$ & $-11.9(6)$ \\
\hline RCA & $-10 / 4(4)$ & $-8.9(3)$ & $-7.7(3)$ & -6.0 (2) $P=\mathbf{0 . 0 0 2}$ & $-6.2(2)$ \\
\hline \multicolumn{6}{|l|}{ Ecc Endo } \\
\hline Global & $-24.0(4)$ & -13.7 (5) $\mathbf{P}=\mathbf{0 . 0 0 4}$ & $-18.0(4)$ & -16.1 (6) $P=0.001$ & -11.8 (5) $\mathbf{P}=\mathbf{0 . 0 3}$ \\
\hline Basal & $-23.8(5)$ & $-17.2(5) \mathbf{P}=\mathbf{0 . 0 4}$ & $-171 .(4)$ & -13.8 (6) $\mathbf{P}=\mathbf{0 . 0 0 7}$ & -12.2 (5) $\mathbf{P}=\mathbf{0 . 0 5}$ \\
\hline Mid & $-23.0(5)$ & $-18.8(5)$ & $-15.2(5)$ & -13.5 (6) $\mathbf{P}=\mathbf{0 . 0 0 4}$ & -10.4 (4) $\mathbf{P}=\mathbf{0 . 0 4}$ \\
\hline Apical & $-27.1(7)$ & $-11.3(6) \mathbf{P}=\mathbf{0 . 0 1 4}$ & $-24.5(8)$ & $-24.5(10)$ & $-14.4(9)$ \\
\hline LAD & $-26.9(6)$ & -10.7 (6) $\mathbf{P}=\mathbf{0 . 0 2}$ & $-22.1(5)$ & -18.9 (8) $\mathbf{P}=\mathbf{0 . 0 0 4}$ & $-12.2(5)$ \\
\hline LCX & $-25.0(7)$ & $-19.0(6)$ & $-17.3(3)$ & $-21.7(8)$ & $-14.3(6)$ \\
\hline RCA & $-20.7(4)$ & $-15.6(6)$ & $-14.4(4)$ & $-11.8(7)$ & $-11.2(5)$ \\
\hline
\end{tabular}

doi:10.1186/1532-429X-13-S1-P131

Cite this article as: Shafi et al:: Quantitative assessment of global and regional strain in relation to infarct size in patients with myocardial infarction. Journal of Cardiovascular Magnetic Resonance 2011 13(Suppl 1): P131.

Submit your next manuscript to BioMed Central and take full advantage of:

- Convenient online submission

- Thorough peer review

- No space constraints or color figure charges

- Immediate publication on acceptance

- Inclusion in PubMed, CAS, Scopus and Google Scholar

- Research which is freely available for redistribution

Submit your manuscript at www.biomedcentral.com/submit
C Biomed Central 